\section{Yorick's luck}

Mark Ridley

Sex and Death in Protozoa: The History of an Obsession. By Graham Bell. Cambridge University Press: 1989. Pp. 199. $£ 25, \$ 44.50$.

For half a century, from about 1880 to 1930 , many biologists were busily trying to maintain perpetual, immortal cultures of protozoans. All the leading scientific nations contributed to this great research effort: it began with Germans, such as Engelmann and Weismann, was continued in France by Maupas and Balbiani, and the torch was passed, for the final phase, to such industrious Americans as Calkins, Jennings and Woodruff.

The main, though by no means the only, research organism was Paramecium. The work concentrated on 'isolate' cultures: no one doubted that 'mass' cultures with millions of protozoans could be kept going practically indefinitely. In an isolate culture, an individual protozoan is isolated and used to seed a new culture; from this culture, after a day or so, another individual is isolated and used in turn to seed a second culture, and so on. Some biologists claimed these cultures could be immortal; others that they inevitably senesced, as the organisms produced abnormalities and their fission rate declined.

There was another question. Could a senescent culture, if conditions were altered to permit sexual conjugation, be 'rejuvenated'? Again, some said yes, others no. And, according to Graham Bell, "the problem was never resolved; after fifty years it was simply abandoned". The body - or corpse - of that work has long since been buried under the dust of scholarship.

Bell's new book is a piece of graverobbery, for he has read this old literature and has brought it to bear on our modern interests in the evolution of senescence, repair mechanisms, recombination and, particularly, sex. Incidentally, he was able to solve the old problem itself. $\mathrm{He}$ has located 75 experiments that can be analysed statistically. Of these, 65 show a negative relation of fission rate and time: the results (almost unknown to the experimenters) overwhelmingly confirm that isolate protozoan cultures do indeed 'senesce'. Without sex, a protozoan lineage will become extinct after a few hundred generations.

Bell also finds that sexual conjugation does revive a senescent lineage. The best experiment was by Calkins, who isolated conjugating couples from an otherwise asexual lineage of Uroleptus mobilis. Lines bred from the conjugants consistently out-lasted the original parental lines.
Bell calls this "one of the more important experimental results of biology". It is certainly one of the more agreeable. Protozoans (in which, it should be recalled, sex is separate from reproduction) carry out conjugation in order to prolong their lives.

A number of other provocative results are conjured out of these old papers. But Bell's main concern is with the significance of the two principal results. In a chapter on senescence, he argues that decay of protozoan cultures is fundamentally different from senescence in multicellular organisms. He accepts the theory of Medawar and G.C. Williams, according to which animals like ourselves senesce because selection favours increased early survival at the expense of survival later in life. Bell believes that senescence in protozoan cultures is caused instead by the accumulation of deleterious mutations.

Reproduction in isolate cultures is parthenogenetic, by binary fission, and the accumulation of mutations thus illustrates one of the main theories of sex the one known as 'Muller's Ratchet'. Sex, if this theory is right, exists to purge the DNA of the deleterious mutations that tend to build up in an asexual lineage. In Bell's neat image, sex is an exogenous repair mechanism. It is impossible, in a population of asexual organisms, to generate an organism with less than the present minimum number of deleterious mutations. This minimum number, moreover, tends to increase through time. If all the organisms with the current minimum number of mutations by chance all die without issue, the new minimum number

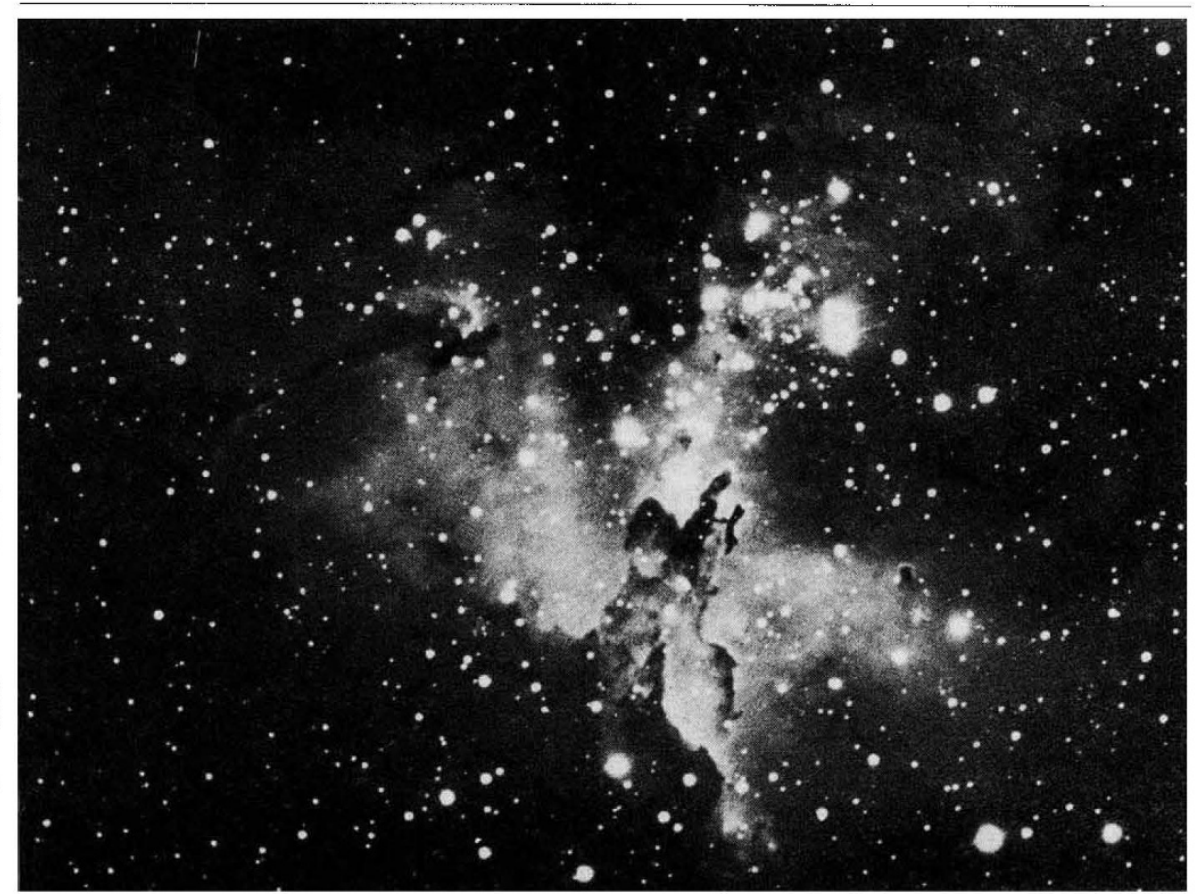

The Eagle Nebula (M16) - a detail taken from one of the prints in Precision Astrophotography, a set of 10 ( $11 \times 14$ inch) photographs by Kim Zussman. The collection, together with two others in colour (The Finest Galaxies and Deep-sky Photography), is published by Kalmbach, 21027 Crossroads Circle, PO Box 1612, Waukesha, Wisconsin 53187, USA. Price is $\$ 16.95$ per set.

will increase. Muller's Ratchet will have turned. In a sexual population, an organism with fewer deleterious mutations can be generated by recombination, and the number of mutations therefore does not tend to increase. According to Bell, "the history of isolate cultures or protozoans provides as comprehensive an illustration of the Ratchet as one could wish for".

$\mathrm{He}$ is probably correct, but his interpretation is not so well established as he concludes. Other theories of sex could account for the same observations. A popular theory at present suggests that sex exists because of the coevolutionary relations of parasites and hosts. It is perfectly possible that, in an isolate culture, parasites to which that particular protozoan lineage lacks resistance genes will build up. Outcrossing could then introduce the resistance mechanisms, and rejuvenate the culture. Other phenomena, such as the spindly taxonomic distribution of asexual reproduction, which Bell explains by the Ratchet, can also be explained by most other theories of sex.

These are minor criticisms. This old and forgotten literature has turned out, in Bell's hands, to contain some unexpectedly suggestive results for more modern and fundamental problems. He has evidently enjoyed exhuming the 'musty volumes'. His book is concise, elegant, non-technical and (that rare thing) a thoroughly good read.

Mark Ridley is in the Department of Zoology, University of Cambridge, Downing Street, Cambridge CB2 $3 E$, UK. 